\title{
Familiarity or Conceptual Priming: Event-related Potentials in Name Recognition
}

\author{
Georg Stenberg ${ }^{1}$, Johan Hellman², Mikael Johansson ${ }^{2}$, \\ and Ingmar Rosén 2
}

\begin{abstract}
Recent interest has been drawn to the separate components of recognition memory, as studied by event-related potentials (ERPs). In ERPs, recollection is usually accompanied by a late, parietal positive deflection. An earlier, frontal component has been suggested to be a counterpart, accompanying recognition by familiarity. However, this component, the FN400, has alternatively been suggested to reflect a form of implicit memory, conceptual priming. The present study examined the ERP components of recognition memory using an episodic memory task with a stimulus material consisting of names, half of which were famous. Along a different dimension, the names varied in how rare or common they were. These dimensions,
\end{abstract}

\section{INTRODUCTION}

Recognition is a deceptively simple form of memory. In the layman's view, it is a matter of comparing the present stimulus to stored images in memory and finding a match. Yet, we frequently come across the experience of knowing a match is there, despite having searched memory unsuccessfully. A face can look hauntingly familiar, but defy all our attempts to match it with a name. Experiences such as these have inspired the view that recognition consists of two processes, in at least partial independence of each other: familiarity and recollection. We can have one without the other, such as when we fail to recollect where we met the person with the familiar face (Mandler, 1980).

In explaining this phenomenon, researchers have chosen two different paths (Yonelinas, 2002). Dual-process theories have proposed that recognition makes use of two qualitatively different types of information. Familiarity is based on a nonspecific computation of memory strength, subjectively accessible as a general feeling of knowing. Recollection, on the other hand, makes use of more detailed information, is often specific about time and place, and helps reconstruct the episode when the stimulus was encountered.

\footnotetext{
${ }^{1}$ Kristianstad University, Sweden, ${ }^{2}$ Lund University, Sweden
}

frequency and fame, exerted powerful effects on memory accuracy, and dissociated the two recognition processes, such that frequency gave rise to familiarity and fame fostered recollection, when the receiver operating characteristics data were analyzed with Yonelinas' dual-process signal detection model. The ERPs corresponded fully to the behavioral data because frequency affected the frontal component exclusively, and fame affected the parietal component exclusively. Moreover, a separate behavioral experiment showed that conceptual priming was sensitive to fame, but not to frequency. Our data therefore indicate that the FN400 varies jointly with familiarity, but independently of conceptual priming.

Single-process theories, in contrast, do not deny that recognition can give rise to a spectrum of experiences, from the vaguest to the most detailed, but claim that they can all be placed on the same continuum. Thus, no separate kind of information is accessed to dignify a vague feeling of familiarity into full-blown recollection, only more of the same, held with more confidence. Single-process theories have often been articulated using the concepts of signal detection theory, and quantitative models (Clark \& Gronlund, 1996) have been remarkably successful in explaining a variety of memory phenomena within this framework. This parsimony is not given up lightly, and the burden of proof rests upon dual-process theories to justify claims to the contrary.

Familiarity is assumed to operate quickly, giving a general estimate of the similarity between an item and the contents of memory (Hintzman \& Curran, 1994). Recollection is the more effortful of the two. If successful, it gives access to a stored representation of an earlier event in its full context, allowing a re-experience of the episode. It is often thought that familiarity is a graded and stochastic process, well described by signal detection theory. More disputed is the contrasting claim that recollection is an all-or-none phenomenon, described by high-threshold theory (Parks \& Yonelinas, 2007; Wixted, 2007).

Ways of separating the two recognition processes using behavioral methods have been developed. The 
remember/know procedure lets the participant characterize her subjective experience by prompting her with a follow-up question after each affirmative recognition decision: "Do you remember seeing the word, or do you just know it was there?"(Gardiner \& RichardsonKlavehn, 2000) Another method takes advantage of the observation that recollection almost always is accompanied by a high degree of confidence, whereas familiarity can run the whole gamut, from conviction to guessing. The method, which has been devised by Andrew Yonelinas (2001a), is based on data gathered in the receiver operating characteristics (ROC) paradigm, that is, a recognition task where decisions are made on a (usually) 6-point scale, running from "Quite sure it is new" to "Quite sure it is old." A model is fitted to the accumulated data, in which a familiarity component accounts for the signal detection share of the responses, and a recollection component accounts for the affirmative responses at the high-confidence end of the scale.

Experimental manipulations, such as a source monitoring task, can expose the kind of contextual knowledge that recollection is privy to. Hence, conditions in which source judgments are correct can be assumed to rely on recollection. Further, manipulations of processing levels at encoding can produce the desired type of memory (Rugg et al., 1998): Deep encoding makes recollection more likely, and shallow encoding makes it unlikely, leaving familiarity as the only resort.

The great interest in the two processes of recognition has been staked on the claim that different neural structures are involved. Based on extensive experimentation with animal analogues of familiarity and recollection, key roles have been assigned to the perirhinal cortex for the former and to the hippocampus for the latter (Aggleton \& Brown, 2006; Brown \& Aggleton, 2001).

The human neuroimaging and neuropsychology data have recently been reviewed (Skinner \& Fernandes, 2007). The relevant fMRI studies show activation patterns compatible with, but not restricted to, the expected areas of the medial-temporal lobe. Areas of prefrontal and parietal cortices as well as content-specific sensory regions are also brought into play by recognition processes. Recollection produces activation that it shares with familiarity, and moreover, brings additional regions into a more extensive and possibly a more coherent network (Skinner \& Fernandes, 2007). The clinical data show areas in which lesions harm recollection but not familiarity (Yonelinas et al., 2004), but the opposite pattern has been harder to find. Recently, however, a double dissociation has been discovered, in which the volume of the hippocampus correlated with recollection in a group of elderly people, and, importantly, the volume of the entorhinal cortex correlated with familiarity (Yonelinas et al., in press).

Event-related potentials (ERPs) have provided converging evidence (recently reviewed by Rugg \& Curran,
2007). The proposed two processes of recognition have been associated with two characteristic electrophysiological signatures, which can be seen as components in old-new difference waveforms. The slower process of recollection gives rise to a relatively late (400-900 msec) component (Rugg \& Yonelinas, 2003) with a parietal distribution, often with a left-over-right asymmetry. Behavioral evidence gathered jointly with ERPs testify in favor of the connection with recollection. Thus, the component is sensitive to levels-of-processing manipulations (Paller, Kutas, \& McIsaac, 1995), and larger for items eliciting "remember" rather than "know" responses (Curran, 2004). It responds to source memory tasks and associative recognition even more than to item memory. It is drastically curtailed by lesions to major memory areas in the brain such as the medial-temporal lobe (Duzel, Vargha-Khadem, Heinze, \& Mishkin, 2001). From what is known through other methods, there are reasons to believe that the hippocampus takes a crucial part, through interactions with the cortex, in the encoding and retrieval of recollected memories (Norman \& O'Reilly, 2003). The parietal scalp distribution is thought to reflect mainly the cortical contributions to this exchange.

Following the parietal old-new positive deflection, one additional component may appear under some conditions. It is a late posterior negativity (Johansson \& Mecklinger, 2003), which appears with an onset latency of approximately $800 \mathrm{msec}$ and a mainly parietal distribution. It is elicited in relatively demanding tasks where the participant is expected to determine in what context the stimulus was encountered (e.g., Johansson, Stenberg, Lindgren, \& Rosen, 2002; Cycowicz, Friedman, \& Snodgrass, 2001) or is otherwise made to monitor his actions closely for errors (Curran, DeBuse, \& Leynes, 2007; Herron, 2007; Nessler \& Mecklinger, 2003).

The large parietal component was apparent in early stages of research into old-new ERP effects. A component corresponding to familiarity is a more recent finding (Mecklinger, 2006; Curran, 2000). It is called the FN400, or the mid-frontal old-new effect, because it is seen as a frontal, positive-going modulation of a negative component with a peak around $400 \mathrm{msec}$. A number of characteristics have made an association with familiarity seem likely. It is elicited by lures that are closely similar to studied items, such as the word "horses" when "horse" has been studied, or a mirror-reversed version of a studied image (Mecklinger, 2006; Curran \& Cleary, 2003; Curran, 2000). Unlike the parietal effect, the FN400 seems sensitive only to the general similarity, not to the finer distinctions between targets and lures, even when instructions emphasize them. Its early latency (300-500 msec) is compatible with a fast-acting process. Further, it covaries with response confidence, as would be expected of a continuous signal-detection process (Woodruff, Hayama, \& Rugg, 2006).

However, another interpretation has been proposed, which associates the FN400 not with familiarity, but with 
conceptual priming (Paller, Voss, \& Boehm, 2007). Paller, Voss, and Boehm (2007) observed that many of the tasks in which the FN400 has been produced contain an element of conceptual overlap between study and test. For example, the lures with altered plurality in Curran's (2000) study were probably conceptually primed in the study phase to roughly the same degree as preservedplurality targets. This priming could produce the attenuation of an N400 that has become known as the FN400. Supporting this alternative explanation, the authors adduced studies with negative findings where the familiarity hypothesis would have predicted an FN400 effect. A case in point comes from a study (Yovel \& Paller, 2004) of face recognition with a contextual memory component. The task was to learn an occupation presented auditorily along with each face. Faces that were recognized without retrieval of the occupation were said to be recognized by familiarity alone. These faces produced no FN400, only the parietal old-new effect, but with lower amplitude than the recollected face-occupation pairs. Instead, in a study where conceptual priming was measured, it was found to correlate with a frontal old-new effect, whereas a measure of explicit memory correlated with a parietal effect (Voss \& Paller, 2006).

A prediction can be deduced from Paller et al.'s position, viz. that items without conceptual content produce no FN400, no matter how familiar they may be. The prediction applies to pseudowords and nonfigurative drawings, among other things. Unfortunately, the data hitherto are not very clear on this point. Completely meaningless stimuli are hard to find, considering that meaning is in the eye of the beholder. When pseudowords and quasi-drawings do give rise to FN400s and have been claimed as evidence against the conceptualpriming interpretation, their meaninglessness has been contested (Paller et al., 2007; Rugg \& Curran, 2007).

\section{Purpose}

Our aim was to dissociate recollection from familiarity behaviorally to permit study of the electrophysiological signatures of the two processes in retrieval. As a tool, we use a stimulus set of names that we have used before for the same purpose (Stenberg, Hellman, \& Johansson, in press). The idea is to let participants' previous (preexperimental) acquaintance with the material determine the way in which it will be remembered. The material varies along two dimensions that we have found to predispose toward familiarity and recollection. We will verify that they do so in the present sample also, using Yonelinas' method of analyzing ROC data, the dualprocess signal detection (DPSD) model.

The stimulus material consists of names, a common object for semantic memory (and with age, a proverbial source of memory lapses). The set of names we used (first name plus last name, all Swedish) vary in media exposure, some of them being names of well-known celebrities with frequent appearances in news media, others being completely incognito. Along a different dimension, they also vary in how rare or common they are, some being shared by many namesakes, others being unique. Examples of famous, rare names (in English-speaking countries) would be Barack Obama and Gwyneth Paltrow. Common, famous names are, for example, Jessica Simpson and Will Smith, and common nonfamous names are Tom Williams and Jane Wilson. Rare, nonfamous names would be, for example, Sebastian Weisdorf and Guido Bagnaschi.

Elsewhere, we have proposed that these two dimensions correspond to two types of semantic memory (Stenberg et al., in press). In the present context, we are concerned with how they interact with episodic memory, and in particular, we suggest a special relation with the dual processes of recollection and familiarity.

Famous names are prone to be recalled with a rich context of remembered associations. Because of the prior knowledge we possess about the person who bears it, the name is readily woven into a web of associated facts, thoughts, and images that can serve as aids in future retrieval attempts. This aspect of the memory for famous names lets it provide material for recollection.

Familiarity, the other major process of recognition memory, is what we suggest to be related to name frequency, that is, the probability of coming across the name in daily life. A common name, such as John Smith, will, no doubt, sound familiar on first presentation. But it will not be easily remembered, being subject to interference from many other, similar instances. The sense in which we suggest that name frequency operates on memory by familiarity is quite the opposite: Uncommon names are recognized by the sense of familiarity they evoke. This conjecture, the familiarity increment hypothesis (Mandler, 1980), will be elaborated a little further ahead.

In brief, we expect a relation to hold between properties of our stimulus material and the processes they set in motion in a memory experiment. The dimensions of celebrity and frequency are varied orthogonally in our set of names, whose validity has been checked by searches of the Internet. We counted the number of times each name was used on national mass media web sites; we included a sample of morning newspapers, tabloids, and television networks. The number served as a measure of celebrity. Acting as proxy for name frequency was the number of persons bearing the name listed in a national, Web-accessible telephone directory. In an earlier study (Stenberg et al., in press), we have validated these qualities in our name database, showing good agreement between participant ratings and the Internet search statistics. And crucially, we demonstrated strong, independent effects of both variables on episodic recognition performance. Famous names were better remembered than nonfamous ones, infrequent 
names better than frequent ones. To these findings we now wish to add an examination of the brain processes involved. (Some aspects of the behavioral data from the present experiment were reported as Experiment 4 in Stenberg et al., in press).

\section{Hypotheses}

We expect application of the Yonelinas model to reveal the extent to which different categories of names are recognized by recollection or familiarity. We expect a mapping between recollection and celebrity in our material, such that famous names are recollected more often than nonfamous ones. Similarly, we expect a mapping between familiarity and frequency, such that infrequent names seem more familiar at the time of test than frequent ones (in a sense to be explained shortly).

Yonelinas' model has been deployed in studies of ordinary item recognition as well as of associative recognition (Yonelinas, 1997), of source memory (Yonelinas, 1999), and of amnesic patients (Yonelinas, Kroll, Dobbins, Lazzara, \& Knight, 1998). It has been validated against other methods of separating dual processes, such as Jacoby's process dissociation procedure (Yonelinas, 1994) and the remember/know procedure (Yonelinas, 2001b). However, to our knowledge, the model has not been applied previously in direct connection with ERP measures of recollection and familiarity.

Our expected mapping between recollection and celebrity, on the one hand, and familiarity and frequency, on the other, may turn out to involve full, partial, or no overlap between the two processes. As a working hypothesis, we choose the simpler alternative and assume a double dissociation. In other words, we assume a relation between recollection and celebrity alone, on the one hand, and familiarity and frequency alone, on the other.

\section{The Familiarity Increment Hypothesis}

The ways in which accuracy in a memory test is affected by a name's frequency deserve working out in some detail. We will use the term memory strength to denote the dimension underlying memory decisions in global matching models of memory (Murdock, 1993; Gillund \& Shiffrin, 1984; Hintzman, 1984). Memory strength arises as a function of comparisons performed against all stored memory items. Therefore, obviously, an infrequent name has lower memory strength at the outset, that is, when presented for the first time in the experiment than a frequent name. In the test phase, a studied infrequent name has gained something in strength as a result of the study episode, but it may still be lower in strength than a studied, or even an unstudied, frequent name. However, the testee is likely to make his decision based on a Bayesian mechanism, taking into account the prior memory strength, given the frequency of the name. In other words, a rare name has low a priori memory strength, and in view of this, the participant may very well decide that a tiny elevation of strength is a telltale sign of its being studied. In reference to the word frequency effect on recognition, Mandler (1980) wrote in his seminal paper (p. 267): "On the basis of familiarity alone, the best explanatory candidate is an incremental explanation; that is, the additional presentation produces a larger relative increment for low than for high frequency words." This view is also in agreement with the BIC (binding of items and context) model of medial-temporal lobe functioning (Diana, Yonelinas, \& Ranganath, 2007) and the Gatekeeper model (Fernandez \& Tendolkar, 2006), according to which activity in the perirhinal cortex is high when encoding new and unfamiliar objects, and correspondingly low when recognizing the familiar.

This Bayesian likelihood mechanism is the mainspring in most modern explanations of the mirror effect. In the present context, it provides the explanation for better recognition of rare than of common names. For example, in the memory model REM (Shiffrin \& Steyvers, 1997), likelihood ratios are formed for each feature and combined into overall odds for the item being old versus the item being new. In this process, unusual features contribute heavily because of their diagnosticity. The probability of an unusual name matching even a faint memory trace by chance is low, and the memory mechanism takes this into account automatically when deciding that the item is old. It is worth emphasizing that this Bayesian weighing of the evidence takes place for each and every item, as part of the standard processing of stimuli. Thus, it is not a recourse of extra deliberation, consciously chosen to resolve a temporarily suspended judgment on a thorny issue. The mechanism deciding about memory strength acts quickly and automatically, corresponding to the familiarity component of twoprocess theories. And that is the reason we expect the infrequent names to be better recognized than frequent names, by virtue of their familiarity, paradoxical as this may sound.

\section{ERP Hypotheses}

In the test phase ERPs, we anticipate old-new differences in the early stages (300-500 msec) as a positive deflection over frontal areas, the FN400 effect. This effect, we hypothesize, will be greater for the types of stimuli that are primarily recognized on the basis of familiarity (i.e., the infrequent names).

We also wish to compare the conditions in which we find an FN400 with the conditions that produce conceptual priming. If the conceptual priming hypothesis for the FN400 is tenable, conditions conducive to priming should also produce the FN400. If, on the other hand, the familiarity hypothesis is right, conditions that give behavioral signs of recognition by familiarity should also produce the FN400. 
In a later stage (400-900), we expect to see large oldnew differences as a positive deflection, maximal over parietal areas. If our hypothesis is correct, it will be greater for famous names than for nonfamous ones because the former can be recollected more readily.

A late posterior negativity can be expected for names that are ambiguous as to source. In our material, many of the names are well known pre-experimentally, particularly the names of celebrities, but also the more common names. These stimuli necessitate close scrutiny to determine whether the name was recently seen in the media or in the experiment. This is the type of task that has been known to produce the late posterior negativity (Johansson \& Mecklinger, 2003).

\section{EXPERIMENT 1}

\section{Methods}

\section{Participants}

Twenty-four students at Lund University (14 women) participated in the first experiment, which was conducted in a laboratory at the University Hospital. Each participant was tested individually during an approximately 1-hr-long session, and received a cinema ticket voucher in compensation. Age of participants averaged 24.8 years, with a range of $19-42$ years.

\section{Materials}

A set of 288 Swedish names was constructed. Names were either selected from the set of those at the time (early 2006) popular in the media, or combined (first name plus last name) using frequency tables provided by the national census bureau, Statistics Sweden. The experimenters, when constructing the stimulus material, judged each name as either famous or nonfamous, and either frequent or infrequent. Seventy-two names of each of four types were selected. Examples of famous names were: Greta Garbo, Ingmar Bergman, and Björn Borg. The set is available at: www.stenberg.ys.se/Projects/ Names/Names.htm.

To verify the judgments, names were checked for frequency by looking up each name in the Swedish nationwide telephone directory, and noting the number of hits. This number was log transformed, and used as the variable frequency, which was dichotomized into frequent and infrequent.

Similarly, celebrity was checked by making site-specific lookups via the Google search engine. Each name was searched at six Swedish Web sites, affiliated with important media: four national newspapers and two television networks. The number of hits was added across sites, and $\log$ transformed. Finally, the variable was dichotomized into famous and nonfamous.

The number of phone directory hits was unrelated to the number of media hits $(r=.01)$. The set of names is described in more detail in another publication (Stenberg et al., in press), where participant ratings corroborating the Internet data are also given.

The set of names was again validated in Experiment 2 of the present study. In this experiment, binary judgments of fame and frequency were made concerning 128 randomly selected names from the set of 288. Although the task was speeded, agreement with the norms was good. The consistency (intraclass correlation) across 13 participants performing the fame judgment task was .96. Proportion correct was .89, and only two items elicited more wrong than correct responses.

The frequency judgment task had to draw a line arbitrarily between what was to be judged common and rare. Our definition was that names with fewer than 10 bearers in Sweden were to be considered rare. The nine participants in this task showed surprising consistency in this rather contrived task; intraclass correlation was .92. Average proportion correct was .84, and eight items, out of 128 , were judged incorrectly more than half of the time.

\section{Electrophysiological Recording}

The EEG was recorded using tin electrodes in an electrode cap (NeuroScan). Electrodes were placed on the 19 positions of the 10-20 system and referenced to the left mastoid during recording. Additional electrodes were applied to monitor vertical eye movements (VEOG; above and below the left eye), and horizontal eye movements (HEOG; outside the outer canthi). One electrode was applied to the right mastoid and recorded for use in later re-referencing. Electrode sites Fpz and Oz were interpolated from adjacent electrodes.

Amplifiers were set to accept frequencies from 0.1 to $30 \mathrm{~Hz}$, and digitization was performed at a rate of $250 \mathrm{~Hz}$. The data were saved continuously to disk during the session for later off-line processing. The files were visually inspected, and EEG stretches with large artifacts were rejected.

From the continuous EEG files, a template for a typical blink artifact was computed, and corrections for blinks were made to the EEG channels using a regression approach implemented in the NeuroScan software. All EEG channels were re-referenced digitally to an average of the left and the right mastoid. The files were segmented into epochs, consisting of $300 \mathrm{msec}$ prestimulus and $1500 \mathrm{msec}$ poststimulus, and the epochs were baseline-corrected by subtraction of the prestimulus average. An artifact rejection algorithm discarded epochs where any EEG channel deviated from baseline by more than $150 \mu \mathrm{V}$. The files were digitally low-pass filtered with a cutoff of $15 \mathrm{~Hz}$ and a rolloff of $48 \mathrm{~dB}$. Behavioral data were used to reject trials with incorrect responses. Finally, averages for the different types of names were formed. 
Most analyses use a $3 \times 3$ grid of the following electrodes; F3, Fz, F4, C3, Cz, C4, P3, Pz, P4 from the 10-20 naming convention.

\section{Procedure}

The pool of 288 names provided stimuli for four blocks, each with 36 studied names and 36 distractors. The four study-test blocks were run consecutively with subjectterminated pauses between them. Assignment of names to study or distractor status was made randomly for each participant, subject to the constraint that each of the four types of names be represented equally in every block.

Stimuli were presented on a computer screen by an E-prime program. Each name was displayed for $2 \mathrm{sec}$ in the study phase, preceded by a 1-sec fixation cross. In the test phase, a name was first shown for $2 \mathrm{sec}$ while responding was disabled (to give ERPs without motor artifacts). Then, a prompt appeared above the name ("Have you seen this name before in the experiment?"), and two response buttons appeared below it, marked "Yes" and "No." A mouse click on either response button terminated this display, which was followed by a new prompt ("How sure are you?") with three response buttons, marked "Quite sure," "Relatively sure," and "Not sure." A maximum of $7 \mathrm{sec}$ was allowed for response selection, but a response terminated the display, usually much sooner.

\section{Data Processing}

Behavioral data. Hit rates and false alarm rates were first computed, using the yes-no responses, and these were used to calculate $d^{\prime}$ as a measure of accuracy. Further, a 6-point scale, ranging from "Quite sure new" to "Quite sure old" was constructed from the responses involving confidence and used for the ROC analysis. Yonelinas' model was applied to the ROC data by a Matlab routine written to fit the model to each individual's responses. For each of the four types of name, a value for $r$, the recollection component, and a value for $d p$, the signal-detection/familiarity component, were determined. The program can be downloaded from www.stenberg.ys.se/Projects/Names/Names.htm.
It works by repeated calls to the standard Matlab function fminsearch, and it applies a set of criteria to judge that a satisfactory solution has been reached. Reliability was assessed by testing with varied random starting values, and was found to be .99.

Statistical issues. When testing effects with more than one degree of freedom in the numerator, the HuynhFeldt correction was applied to the reported probability. The degrees of freedom are given as per before the correction.

\section{Results}

\section{Behavioral Results}

Analysis of the accuracy data proceeded by first computing the conventional signal detection measures, $d^{\prime}$ and $C$, for the four types of names, and submitting them to $2 \times 2$ ANOVAs, with frequency and celebrity as factors. Sensitivity, $d^{\prime}$, showed main effects of both frequency $[F(1,23)=74.30, p<.001]$ and celebrity $[F(1,23)=$ $83.09, p<.001]$. Mean values are shown in Table 1 , as are effect sizes. As expected, fame and rarity made names more memorable, and the two factors contributed about equally, with only a marginal interaction between them $[F(1,23)=4.19, p=.052]$.

The criterion, $C$, was affected only by celebrity $[F(1$, $23)=22.90, p<.001]$ because a balanced (near zero) criterion was maintained for famous names, whereas the nonfamous were judged against a stricter criterion. There was no main effect of frequency, but an interaction between the two factors $[F(1,23)=5.86, p=.024]$.

The dual-process signal detection model. Application of Yonelinas' DPSD model produced a decomposition of ROC curves which is illustrated in Figure 1. Individual ROCs were fitted and the derived averaged values of $r$ (recollection) and $d p$ (the signal detection component) are reported in Table 1 . They were each analyzed in a $2 \times$ 2 ANOVA. On the recollection parameter $r$, celebrity had a marked effect $\left[F(1,23)=30.98, p<.001, \eta_{\mathrm{p}}^{2}=.57\right]$, producing a much higher probability of recollection for famous names. Frequency had no effect $(F<1)$, nor was there any interaction $(p>.2)$.

Table 1. Behavioral Performance in Experiment 1

\begin{tabular}{|c|c|c|c|c|c|c|c|}
\hline & \multicolumn{2}{|c|}{ Infrequent } & \multicolumn{2}{|c|}{ Frequent } & \multicolumn{3}{|c|}{ Effect Size, $\eta_{p}^{2}$} \\
\hline & Nonfamous & Famous & Nonfamous & Famous & Frequency & Celebrity & $\mathrm{Fr} \times \mathrm{Ce}$ \\
\hline$d^{\prime}$ & 2.00 & 2.67 & 1.33 & 2.28 & .76 & .78 & .15 \\
\hline C & 0.25 & 0.11 & 0.37 & 0.06 & .03 & .50 & .20 \\
\hline$r$ & .26 & .49 & .24 & .58 & .03 & .57 & .06 \\
\hline$d p$ & 1.61 & 1.74 & .90 & 1.29 & .55 & .11 & .06 \\
\hline
\end{tabular}


Figure 1. ROC curves for the four types of names. Data points for the summed group ROC are marked by round dots. Blue line indicates model fit. The DPSD model decomposes the ROC into a recollection component, indicated by the horizontal line, and a signal detection component, indicated by the brown curved line. Note the relation between fame and recollection, and between frequency and familiarity. (Because this is a group ROC, the values of the parameters do not coincide exactly with the averaged individual values shown in Table 1, but the pattern across conditions is the same.)

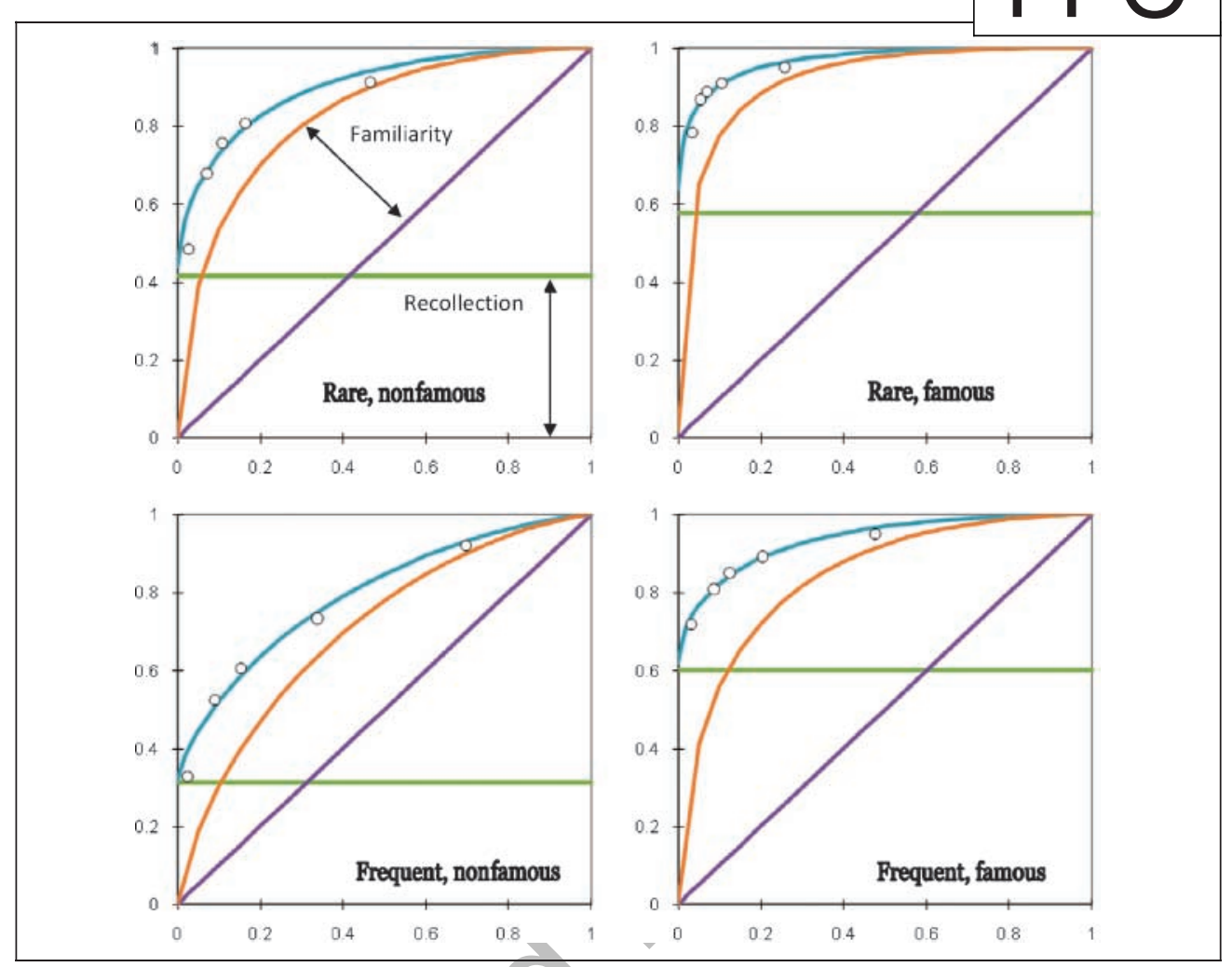

In contrast, the familiarity parameter, $d p$, was dominated by a frequency effect $[F(1,23)=27.79, p<.001$; $\left.\eta_{\mathrm{p}}^{2}=.55\right]$, and celebrity had no effect $[F(1,23)=2.89$, $p>.10]$. There was no interaction $[F(1,23)=1.33$, $p>$.20]. Rare names were more likely to be recognized on the basis of familiarity than were frequent names, as expected by the familiarity increment hypothesis.

Thus, the stimulus qualities frequency and celebrity produced a double dissociation. In terms of Yonelinas' dual-process model, recollection was affected by celebrity alone, and familiarity was affected by frequency alone.

\section{Electrophysiological Results}

Our main interest focused on the old-new effects in the test phase. Having seen indicators of familiarity and recollection, derived from Yonelinas' model, align with the stimulus dimensions of our name stimuli, we wondered whether ERP indicators of recollection and familiarity would do so also. We expected the FN400 to covary with frequency and the parietal old-new effect to covary with celebrity.

Old-new effects. In the test phase ERPs, averages were formed for correctly recognized old names, as well as for correctly rejected new ones, within each of the four types defined by frequency and celebrity. The waveforms are displayed in Figures 2 and 3. The average number of trials contributing to each individual average was: F0C0:
Hits: 27, Correct Rejections: 32; F0C1: H: 32, CR: 34; F1C0: H: 22, CR: 31; F1C1: H: 31, CR: 31 (where, e.g., FOC1 represents low frequency, high celebrity).

An array of nine electrodes, forming a $3 \times 3$ grid over frontal, central, and parietal areas were selected for analysis. The difference waveform was quantified in four intervals: 300-500, 500-700, 700-900, and 900$1100 \mathrm{msec}$. Interest was directed toward the earliest interval for the frontal effect associated with familiarity, and toward the later ones for the parietal effects associated with recollection. Screening for the absence or presence of an overall old-new effect, we tested the intercept effects first. They were reliable in three of the four intervals $[F(1,23)=5.07, p=.034$ in the first; $F(1$, $23)=109.98, p<.001$ in the second; and $F(1,23)=$ $18.49, p<.001$ in the third, but $F(1,23)=1.21$, $n s$ in the last interval].

In the 300-500 msec band, frequency exerted a main effect $\left[F(1,23)=7.76, p=.011, \eta_{\mathrm{p}}^{2}=.25\right]$, modified by an interaction with anterior/posterior position $[F(2$, 46) $\left.=9.77, p=.001 ; \eta_{\mathrm{p}}^{2}=.30\right]$. As will be seen in Figures 2 and 4, there were old-new effects for unusual names only, and these were concentrated frontally, with a gradual decline toward the back $[F(1,23)=11.85$ for the linear trend contrast]. There were no effects involving celebrity in this time band. In other words, the early frontal effect (FN400) was mainly associated with low frequency names.

In the 500-700 msec interval, there were main effects of both celebrity $\left[F(1,23)=4.70, p=.041, \eta_{\mathrm{p}}^{2}=.17\right]$ and, 


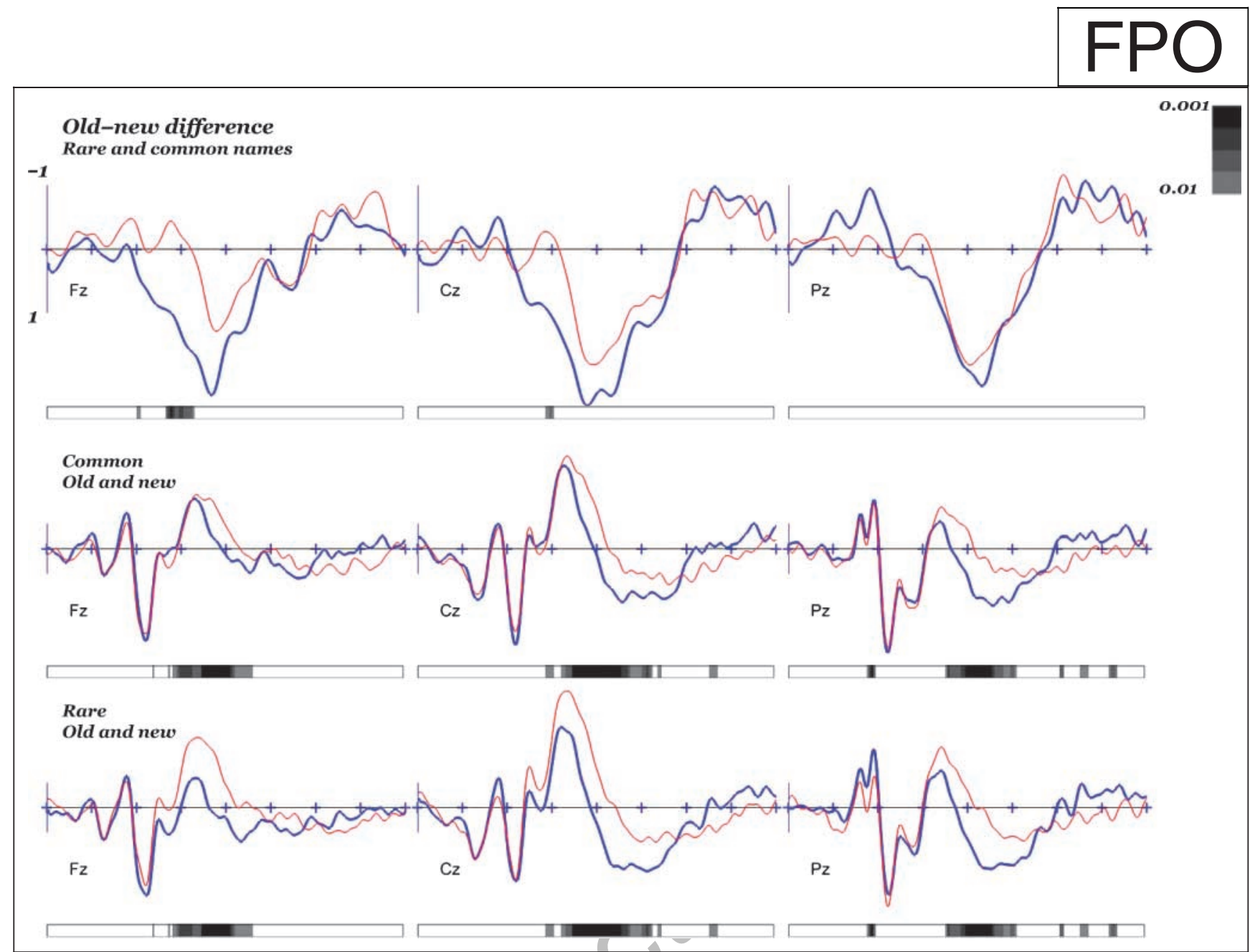

Figure 2. Waveforms in the test phase. Bottom row: Rare names. Thin, red line: new, correctly rejected names. Bold, blue line: old, correctly endorsed names. Second row: Frequent names, new and old. Top row: Difference waves: old minus new. Bold, blue line: rare names; thin red line: frequent names. Electrode locations Fz (frontal), Cz (central), and Pz (parietal) on the midline. In each panel, the bar at the bottom shows the outcome of a sequence of $t$ tests, one every $20 \mathrm{msec}$, of the difference between the two waveforms. Dark bars indicate probabilities <.01. Epoch -200 to $1400 \mathrm{msec}$, a marker each $200 \mathrm{msec}$. Vertical line at the beginning of each trace shows $-1 \mu \mathrm{V}$ (upward) and $+1 \mu \mathrm{V}$ (downward).

marginally, of frequency $\left[F(1,23)=4.20, p=.052, \eta_{\mathrm{p}}^{2}=\right.$ .16]. Their spatial gradients were different, although both interacted with the anterior/posterior position $\left[F(2,46)=5.12, p=.020, \eta_{p}^{2}=.18\right.$ for frequency and $F(2,46)=11.00, p=.001, \eta_{\mathrm{p}}^{2}=.32$ for celebrity]. This proved to be due to opposite linear trends because frequency had its largest effect frontally, $\eta_{p}^{2}=.27$ and a gradual wearing-off toward the back: $\eta_{\mathrm{p}}^{2}=.16$ and $\eta_{\mathrm{p}}^{2}=.02$ for the central and parietal electrodes, respectively. In contrast, the celebrity effect grew stronger at posterior sites: $\eta_{\mathrm{p}}^{2}=.01, \eta_{\mathrm{p}}^{2}=.20$, and $\eta_{\mathrm{p}}^{2}=.33$, for the frontal, central, and parietal sites, respectively. This is illustrated in Figures 2, 3, and 4. The parietal old-new effect was thus most strongly associated with high celebrity names (Figure 5).

In the 700-900 msec interval, no main effects of frequency and celebrity were reliable, and only marginally reliable interactions were found between celebrity and the electrode position factors, $.05<p<.1$, which will not be pursued further. In the 900-1100 msec range, however, the slow posterior wave seen in other high- demand memory tasks arose (Johansson \& Mecklinger, 2003). There was a main effect of celebrity $[F(1,23)=$ $8.82, p=.007]$, as well as a marginal interaction with anterior/posterior position $[F(2,46)=3.53, p=.059]$, and an interaction, Frequency $\times$ Celebrity $[F(1,23)=$ $7.17, p=.013]$. A follow-up test of the parietal row of electrodes showed a main effect of celebrity $[F(1,23)=$ $14.28, p=.001]$, and again an interaction with frequency $[F(1,23)=4.66, p=.041]$, indicating that a late posterior effect accompanied both famous and common names, that is, all types except the infrequent, unheardof names, for which source attribution was not an issue.

Confidence. To assess the effect of confidence on the FN400 and the parietal positive component, trials were sorted into hits and correct rejections, and within each of those categories into high and low confidence responses. Of special interest were the correct rejections. Both theory and earlier investigations (Woodruff et al., 2006) suggest that familiarity is a graded process, correlated with confidence. Recollection is expected to be a 


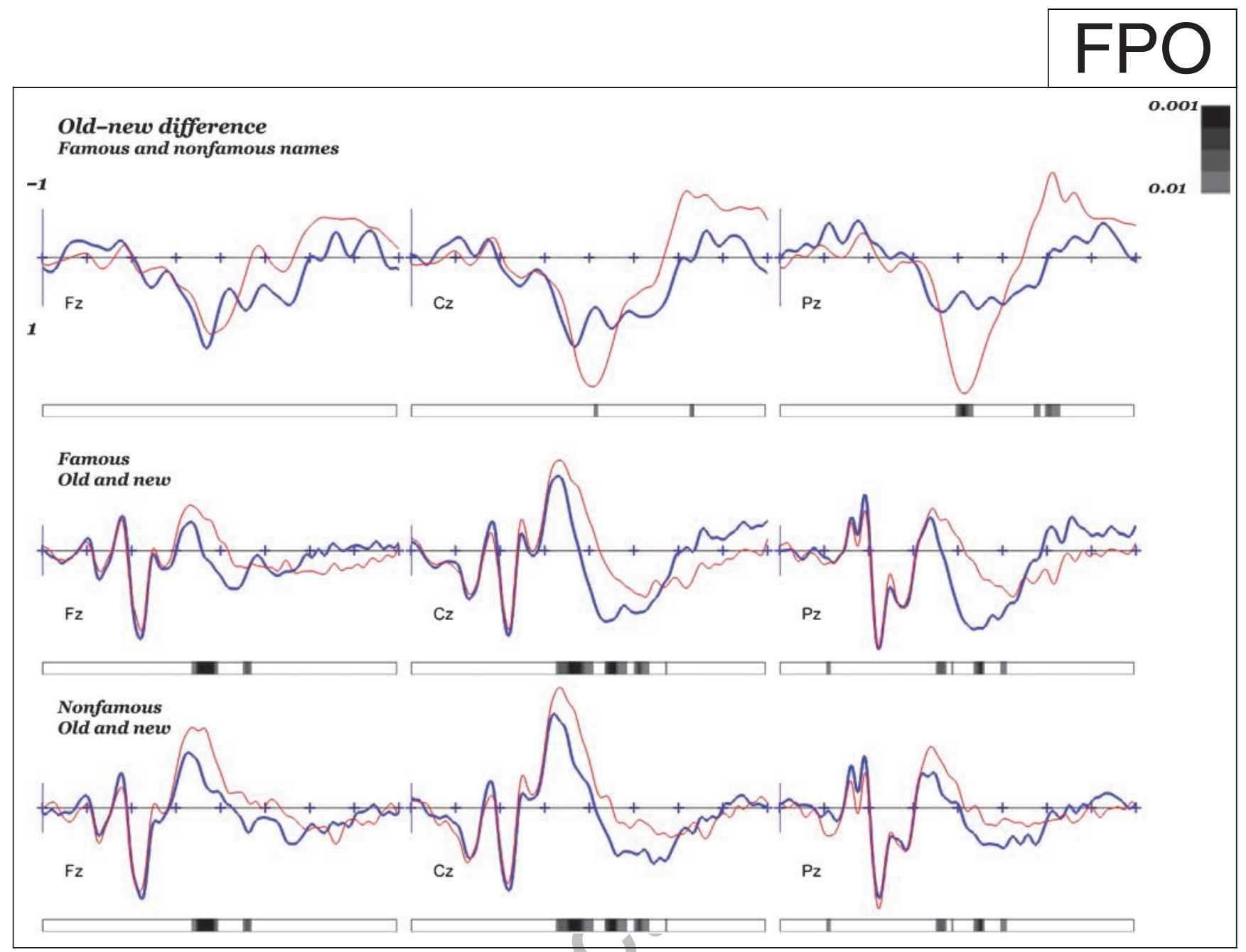

Figure 3. Waveforms in the test phase. Nonfamous (bottom) versus famous (middle row) names. Otherwise as in Figure 2.

thresholded phenomenon, hence, impervious to confidence effects on negative decisions (Curran, 2004). For hits, pairwise $t$ tests showed effects of confidence on both the FN400 (at Fz, 300-500 msec) and the parietal positive component (at $\mathrm{P} 3,500-700 \mathrm{msec}$ ) $[t(22)=2.14$, $p=.043$, and $t(22)=3.87, p=.001$, respectively]. More importantly, correct rejections differed according to confidence only for the FN400 $[t(23)=2.10, p=.047]$, and not for the parietal positive component $[t(23)<1$, ns; see Figure 6].

\section{Discussion}

So far, we have seen how the dimensions of celebrity and familiarity could separate behavioral responses as well as test-phase ERPs along the dividing lines delineated by the two-process theory of recognition. Still, the possibility remains that we have confounded priming with familiarity. We therefore performed a second experiment, in which our stimulus material was used in a task where priming could be measured. The purpose was to see if priming covaried with one of our stimulus dimensions, and if so, which one.

Our expectations are that famous names produce more priming than nonfamous ones. Because they have a clear semantic content, a host of associations can be activated by them, helping to produce facilitation in a renewed encounter. Nonfamous names, on the other hand, evoke few images, especially if they are rare. A faceless name has no semantics and should produce little conceptual priming.

\section{EXPERIMENT 2}

We needed two tasks that could be meaningfully applied to the whole of our stimulus material and could be trusted to activate the conceptual content of the names. Our choice fell upon a frequency judgment task ("Is this a common name?") and a celebrity judgment task ("Is this the name of a famous person?"). Two different tasks, one for the study phase and one for the test phase, were used to avoid measuring just the learning of a fixed stimulus-response mapping.

Responding was speeded, with reaction time as the dependent variable. For one group of participants, the frequency judgment was presented first, in the incidental study phase, and the celebrity task thereafter, at test, when the degree of priming was measured. This will be called the FC condition. For another group (the CF 


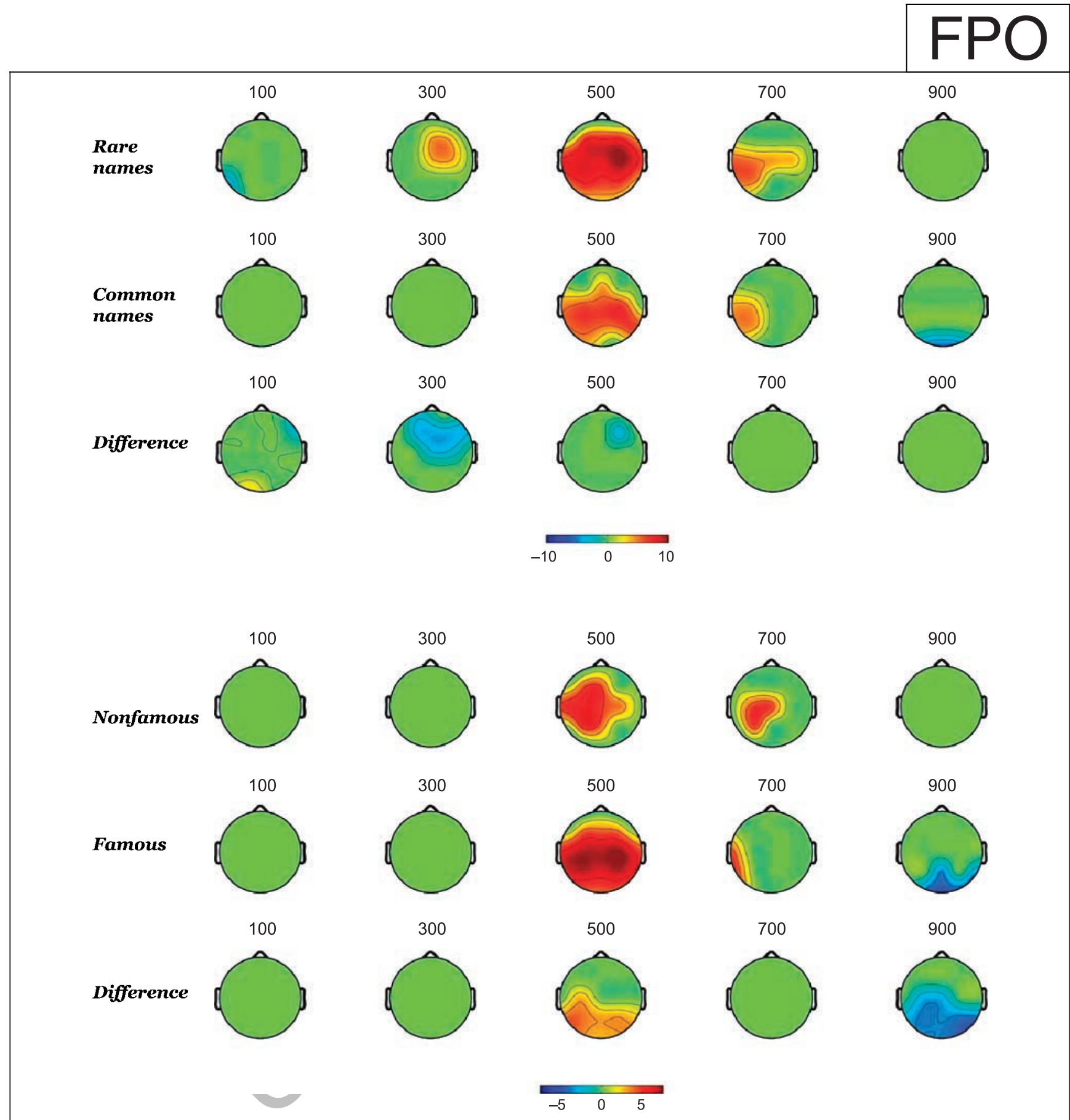

Figure 4. Topographical maps (schematic head seen from above, front upward) showing old-new effects. First and second rows show $t$ values testing whether the old-new effect is different from zero. $t$ Values under a threshold corresponding to $p=.001$ have been set equal to zero. Third row shows $t$ tests of the difference between high and low frequency names, using a threshold of $p=.01$. Rows four to six show similar $t$ tests for high and low celebrity names.

condition), the order was reversed, and priming was measured in the final frequency judgment task. The degree of priming was measured in the second task as the difference in reaction time between re-presented names and new names.

\section{Methods}

The experiment was performed via the Internet, using the Inquisit software (www.millisecond.com). Twenty-two participants were recruited from a pool of volunteer students (two-thirds women, mean age $=25$ years) and university faculty. A set of 64 names was presented in the first task, and the same 64 plus 64 new names were presented in the second task. The assignment of names to either group was counterbalanced across subjects. The whole set of 128 names was randomly selected from our pool of 288, with the restriction that equal numbers of the four types of names be present. Outlier reaction times (with a cutoff at the individual mean $\pm 2.5 S D$ s) were 


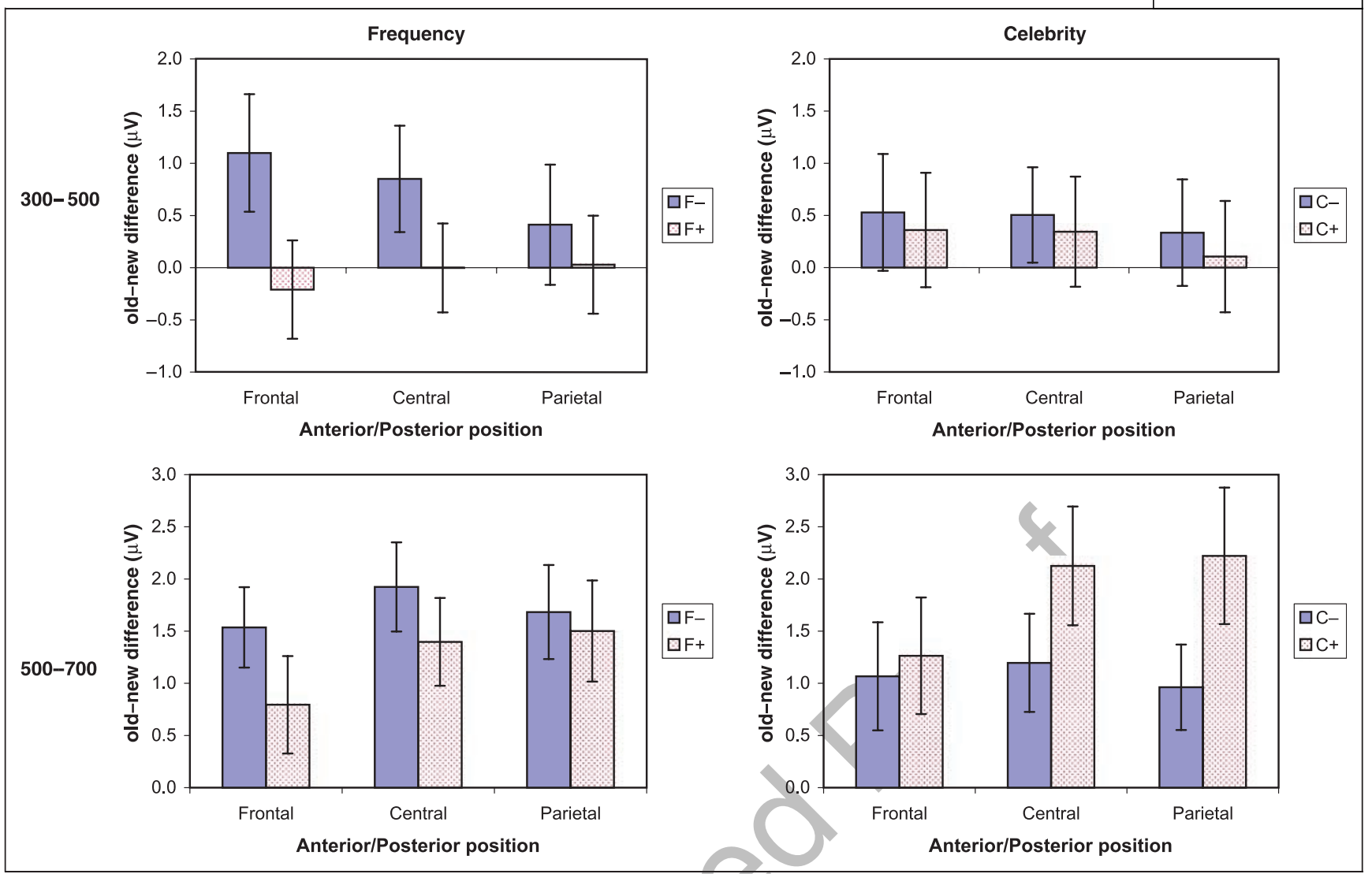

Figure 5. Means and 95\% confidence intervals for the old-new effect in the 300-500 and 500-700 msec intervals. Left panels: high and low frequency names. Right panels: high and low celebrity names. Note the old-new effect in the early interval frontally for rare names (F-). In the later interval there are old-new effects for all types of names, but a maximum can be found parietally for famous names $(\mathrm{C}+)$.

replaced by the cutoff value, and priming scores were formed for the four types of names by subtraction of oldname reaction times from new-name reaction times.

\section{Results and Discussion}

Priming scores (new-name RTs minus old-name RTs) were subjected to a $2 \times 2 \times 2$ (Frequency $\times$ Celebrity $\times$ Task Order) analysis. The main effect of celebrity was significant $[F(1,20)=8.13, p=.01]$ because famous names evinced priming $(M=36 \mathrm{msec}, 95 \% \mathrm{CI}=12-60)$ and nonfamous ones did not. The effect of frequency, and the interaction, was nonsignificant (both $F<1$ ). There was no main effect of task order, but it modified the effect of celebrity through an interaction $[F(1,20)=$ $5.36, p=.031]$. The simple main effect of celebrity was significant in the FC task order $[F(1,12)=26.60$, $p<.001$ ]. In other words, if celebrity was salient when priming was measured, it evinced a large priming effect (61 msec; CI = 23-99, for famous names). On the other hand, when frequency was made salient at test, that is, in the CF task order, neither frequency nor celebrity promoted any priming: all $F \mathrm{~S}<1$ (priming scores for the two task orders are shown in Table 2).

In Figure 7 , priming scores (averaged across task orders) are shown along with FN400 amplitudes (mean of $\mathrm{Fz}$ in 300-500 msec). Conceptual priming was sensitive only to celebrity, and the FN400 only to frequency, that is, they were completely independent.

The present two tasks (speeded frequency and celebrity decisions) used to assess conceptual priming were of the passive verification type. Typical conceptual priming tasks, in contrast, are often production tasks, such as category exemplar generation, and it has been shown that production and verification tasks can yield different results because of different degrees of response competition (Vaidya \& Gabrieli, 2000). However, the recognition task in which the FN400 has been found is also of the verification type. Further, the same perceptual form was repeated in the study and test phases of our conceptual priming experiment, and therefore, it cannot be excluded that perceptual priming also contributed. Against that possibility speaks the fact that there was no priming in the $\mathrm{CF}$ task order, and in any case, our tasks share the perceptual repetition factor with most recognition memory experiments.

\section{GENERAL DISCUSSION}

In summary, we found that our stimulus dimensions dissociated familiarity and recollection. Recollection was accompanied by the expected parietal old-new effect, as 


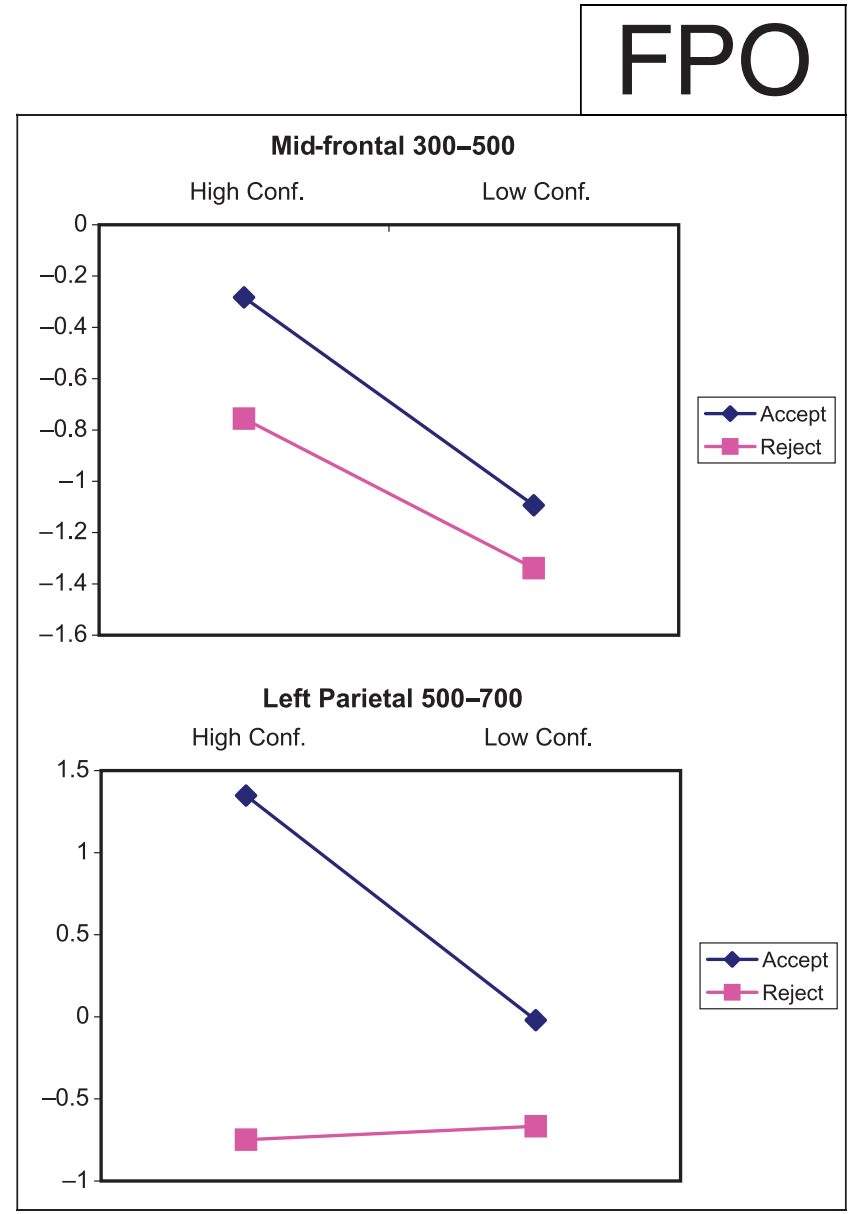

Figure 6. The FN400 and the parietal positive component as a function of confidence and decision (Accept, Reject) in hits and correct rejection. Note that in rejections, FN400 amplitude covaries with confidence, in contrast to the parietal component.

well as a later, posterior negativity, often found in source memory tasks. Familiarity was accompanied by the midfrontal FN400 effect, which, importantly, was orthogonal to conceptual priming.

The behavioral results of this study confirmed the expectation that famous names would be better remembered than nonfamous ones, and that infrequent names

Table 2. Priming Scores in Experiment 2

\begin{tabular}{clcc}
\hline Task Order & \multicolumn{1}{c}{ Type of Name } & $M$ & $S E$ \\
\hline $\begin{array}{c}\text { CF (frequency } \\
\text { task at test) }\end{array}$ & Rare, nonfamous & 7.7 & 39.0 \\
& Rare, famous & -0.8 & 21.9 \\
& Frequent, nonfamous & -4.2 & 18.7 \\
& Frequent, famous & 21.4 & 20.7 \\
FC (celebrity & Rare, nonfamous & -12.6 & 32.5 \\
task at test) & & & \\
& Rare, famous & $78.8^{\mathrm{a}}$ & 18.2 \\
& Frequent, nonfamous & -31.1 & 15.6 \\
& Frequent, famous & $43.2^{\mathrm{a}}$ & 17.2 \\
\hline
\end{tabular}

${ }^{\mathrm{a}}$ Indicates significant priming.

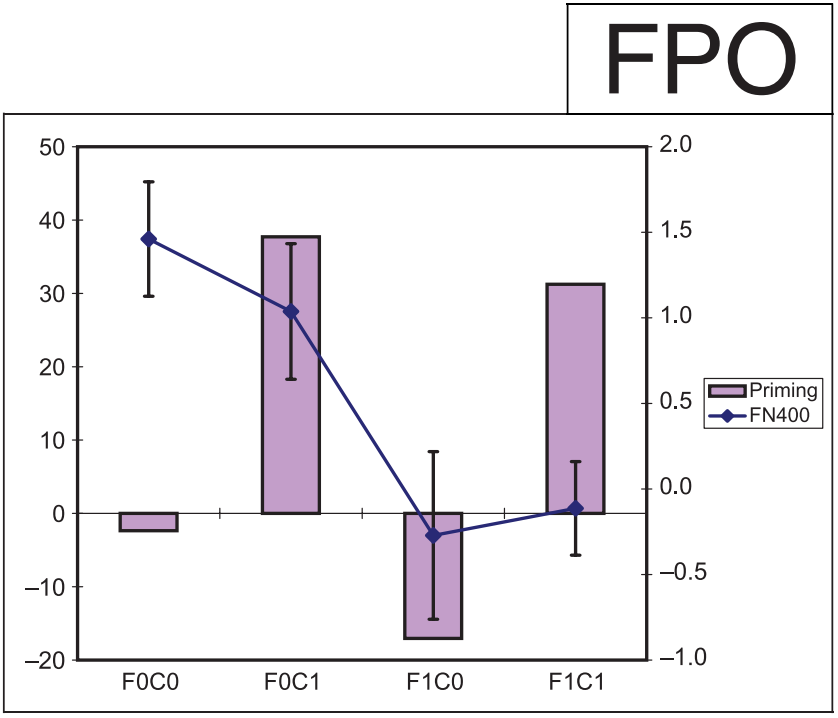

Figure 7. FN400 amplitudes (lines) and conceptual priming scores (bars) across the four types of names, frequency (F) and celebrity (C) to a low (0) or high (1) degree. Error bars equal 1 SEM.

would be better remembered than frequent ones. Crucially, the degree of recollection covaried with the celebrity of the names, and independently, the degree of familiarity covaried with the frequency. In stating this, we rely on analysis according to Yonelinas' DPSD model, which provided parameter values for each name type and each individual. The model rests on the assumption of a high-threshold process underlying recollection, and an equal-variance signal detection process underlying familiarity. Both of these assumptions have been questioned, especially the first. Alternative proposals are afoot, such as single-process, unequal-variance models (Heathcote, Raymond, \& Dunn, 2006), and dual-process models which treat both as continuous signal detection processes (Wixted, 2007; Rotello, Macmillan, \& Reeder, 2004). The viability of the DPSD and its competitors is, at present, a topic under intense investigation (Yonelinas \& Parks, 2007). Our mapping between the name dimensions and memory processes does not rest on the DPSD model alone. In our earlier article (Stenberg et al., in press), we have given several reasons for this mapping, none of which depended upon the assumptions of the DPSD model. We noted that the celebrity dimension affected primarily hit rates, and frequency affected false alarm rates. This pattern is indicative of the workings of two processes (Reder et al., 2000). Moreover, the slopes of the ROCs were affected by celebrity, not by frequency. In toto, the data indicate that the dimensions of the name stimuli align with familiarity and recollection.

The parietal old-new effect proved sensitive to the celebrity of the names. With increasing fame, the network of known facts and associations grows larger, and with this expanded net, the catch increases. The amount of retrieved information grows with contributions from both semantic and episodic memory. It is likely that the amplitude of the parietal effect reflects this abundance (Wilding, 2000). Faced with the task of deciding if the 
retrieved material derives from the experiment proper or from the media, he engages in attempts to reconstruct the context in which the name was recently encountered. This contextual binding effort forms one of the two conditions in which, according to a recent review (Johansson \& Mecklinger, 2003), the late posterior negativity follows the positive parietal old-new effect (see Friedman, Cycowicz, \& Bersick, 2005 for a related view). In our data, famous names evoked the largest positive (500-700) old-new effect, and they also produced the largest negative (900-1100) amplitude modulation. This is consistent with a mechanism that retrieves a mixture of memory facts and episodes, and if necessary, engages in effortful reconstruction of the links from these to their context. With our task, mere quantity of retrieved information was not enough. A definite bond to the context of the experiment was needed to make the information useful. Similarly, task demands for retrieval of special circumstances at encoding, or a "remember" decision have been seen to produce the late, posterior negativity (Mecklinger, Johansson, Parra, \& Hanslmayr, 2007; Wolk et al., 2007).

The mid-frontal effect, the FN400, increased for rare names relative to common names, as did recognition by familiarity. In line with expectations and previous findings, the FN400 proved sensitive in a graded fashion to the strength or confidence (Woodruff et al., 2006; Finnigan, Humphreys, Dennis, \& Geffen, 2002) of both positive and negative decisions, in contrast to the parietal positive component (Curran, 2004), which did not show a graded response in "reject" decisions. The latter finding is predictable from the theoretical position that there can be no below-threshold recollection. Familiarity, on the other hand, can take on any value on a continuous scale.

Our findings are entirely compatible with the view that the FN400 is a reflection of the processes underlying familiarity. The alternative hypothesis, connecting the FN400 with conceptual priming, is difficult to reconcile with the fact that rare names evoked a larger FN400 than common names, and that celebrity made no difference. It would seem that rare names give little opportunity for conceptual processing. Especially the kind of rare names not belonging to a famous person would seem to be the equivalent of pseudowords and nonfigurative doodles, that is, stimuli without meaning. Where there is no conceptual content, there can be no conceptual priming.

Moreover, our data from Experiment 2 are quite unequivocal in showing that priming was sustained by fame, and fame alone. When fame was made salient by the speeded judgment task, famous names produced a large priming effect. When frequency was made salient in the same way, there were no priming effects for any type of name.

Coupled with the completely different relation for the FN400, which was a function of frequency, and frequency alone, these data point unambiguously in one direction. Two independent processes contribute to recognition: familiarity, which is reflected in the FN400, and recollection, which is reflected in two later, parietal components.

\section{Acknowledgments}

This research was supported by grants from the Swedish Research Council to Georg Stenberg ("Det semantiska minnet") and Ingmar Rosén (VR 0084).

Reprint requests should be sent to Georg Stenberg, Department of Psychology, School of Behavioural Sciences, Kristianstad University, SE-291 88 Kristianstad, Sweden, or via e-mail: georg.stenberg@bet.hkr.se.

\section{REFERENCES}

Aggleton, J. P., \& Brown, M. W. (2006). Interleaving brain systems for episodic and recognition memory. Trends in Cognitive Sciences, 10, 455-463.

Brown, M. W., \& Aggleton, J. P. (2001). Recognition memory: What are the roles of the perirhinal cortex and hippocampus? Nature Reviews Neuroscience, 2, 51-61.

Clark, S. E., \& Gronlund, S. D. (1996). Global matching models of recognition memory: How the models match the data. Psychonomic Bulletin \& Review, 3, 37-60.

Curran, T. (2000). Brain potentials of recollection and familiarity. Memory \& Cognition, 28, 923-938.

Curran, T. (2004). Effects of attention and confidence on the

hypothesized ERP correlates of recollection and familiarity. Neuropsychologia, 42, 1088-1106.

Curran, T., \& Cleary, A. M. (2003). Using ERPs to dissociate recollection from familiarity in picture recognition. Cognitive Brain Research, 15, 191-205.

Curran, T., DeBuse, C., \& Leynes, P. A. (2007). Conflict and criterion setting in recognition memory. Journal of Experimental Psychology: Learning, Memory, and Cognition, 33, 2-17.

Cycowicz, Y. M., Friedman, D., \& Snodgrass, J. G. (2001) Remembering the color of objects: An ERP investigation of source memory. Cerebral Cortex, 11, 322-334.

Diana, R. A., Yonelinas, A. P., \& Ranganath, C. (2007). Imaging recollection and familiarity in the medial temporal lobe: A three-component model. Trends in Cognitive Sciences, 11, 379-386.

Duzel, E., Vargha-Khadem, F., Heinze, H. J., \& Mishkin, M. (2001). Brain activity evidence for recognition without recollection after early hippocampal damage. Proceedings of the National Academy of Sciences, U.S.A., 98, 8101-8106.

Fernandez, G., \& Tendolkar, I. (2006). The rhinal cortex: "gatekeeper" of the declarative memory system. Trends in Cognitive Sciences, 10, 358-362.

Finnigan, S., Humphreys, M. S., Dennis, S., \& Geffen, G. (2002). ERP "old/new" effects: Memory strength and decisional factor(s). Neuropsychologia, 40, 2288-2304.

Friedman, D., Cycowicz, Y. M., \& Bersick, M. (2005). The late negative episodic memory effect: The effect of recapitulating study details at test. Brain Research, Cognitive Brain Research, 23, 185-198.

Gardiner, J. M., \& Richardson-Klavehn, A. (2000) Remembering and knowing. In E. Tulving \& F. Craik (Eds.), The Oxford handbook of memory (pp. 229-244). Oxford: Oxford University Press. 
Gillund, G., \& Shiffrin, R. M. (1984). A retrieval model for both recognition and recall. Psychological Review, 91, 1-67.

Heathcote, A., Raymond, F., \& Dunn, J. (2006). Recollection and familiarity in recognition memory: Evidence from ROC curves. Journal of Memory and Language, 55, 495-514.

Herron, J. E. (2007). Decomposition of the ERP late posterior negativity: Effects of retrieval and response fluency. Psychophysiology, 44, 233-244.

Hintzman, D. L. (1984). MINERVA 2: A simulation model of human memory. Behavior Research Methods, Instruments \& Computers, 16, 96-101.

Hintzman, D. L., \& Curran, T. (1994). Retrieval dynamics of recognition and frequency judgments: Evidence for separate processes of familiarity and recall. Journal of Memory and Language, 33, 1-18.

Johansson, M., \& Mecklinger, A. (2003). The late posterior negativity in ERP studies of episodic memory: Action monitoring and retrieval of attribute conjunctions. Biological Psychology, 64, 91-117.

Johansson, M., Stenberg, G., Lindgren, M., \& Rosen, I. (2002). Memory for perceived and imagined pictures-An eventrelated potential study. Neuropsychologia, 40, 986-1002.

Mandler, G. (1980). Recognizing: The judgment of previous occurrence. Psychological Review, 87, 252-271.

Mecklinger, A. (2006). Electrophysiological measures of familiarity memory. Clinical EEG and Neuroscience, 37, 292-299.

Mecklinger, A., Johansson, M., Parra, M., \& Hanslmayr, S. (2007). Source-retrieval requirements influence late ERP and EEG memory effects. Brain Research, 1172, 110-123.

Murdock, B. B. (1993). TODAM2: A model for the storage and retrieval of item, associative, and serial-order information. Psychological Review, 100, 183-203.

Nessler, D., \& Mecklinger, A. (2003). ERP correlates of true and false recognition after different retention delays: Stimulus- and response-related processes. Psychophysiology, 40, 146-159.

Paller, K. A., Kutas, M., \& McIsaac, H. K. (1995). Monitoring conscious recollection via the electrical activity of the brain. Psychological Science, 6, 107-111.

Paller, K. A., Voss, J. L., \& Boehm, S. G. (2007). Validating neural correlates of familiarity. Trends in Cognitive Sciences, 11, 243-250.

Parks, C. M., \& Yonelinas, A. P. (2007). Moving beyond pure signal-detection models: Comment on Wixted (2007). Psychological Review, 114, 188-202; discussion 189-203.

Reder, L. M., Nhouyvanisvong, A., Schunn, C. D., Ayers, M. S., Angstadt, P., \& Hiraki, K. (2000). A mechanistic account of the mirror effect for word frequency: A computational model of remember-know judgments in a continuous recognition paradigm. Journal of Experimental Psychology: Learning, Memory, and Cognition, 26, 294-320.

Rotello, C. M., Macmillan, N. A., \& Reeder, J. A. (2004). Sum-difference theory of remembering and knowing: A two-dimensional signal-detection model. Psychological Review, 111, 588-616.

Rugg, M. D., \& Curran, T. (2007). Event-related potentials and recognition memory. Trends in Cognitive Sciences, 11, 251-257.

Rugg, M. D., Mark, R. E., Walla, P., Schloerscheidt, A. M., Birch, C. S., \& Allan, K. (1998). Dissociation of the neural correlates of implicit and explicit memory. Nature, 392, 595-598.

Rugg, M. D., \& Yonelinas, A. P. (2003). Human recognition memory: A cognitive neuroscience perspective. Trends in Cognitive Sciences, 7, 313-319.

Shiffrin, R. M., \& Steyvers, M. (1997). A model for recognition memory: REM-retrieving effectively from memory. Psychonomic Bulletin \& Review, 4, 145-166.
Skinner, E. I., \& Fernandes, M. A. (2007). Neural correlates of recollection and familiarity: A review of neuroimaging and patient data. Neuropsychologia, 45, 2163-2179.

Stenberg, G., Hellman, J., \& Johansson, M. (in press). The memorability of names and the divergent effects of prior experience. European Journal of Cognitive Psychology.

Vaidya, C. J., \& Gabrieli, J. D. E. (2000). Picture superiority in conceptual memory: Dissociative effects of encoding and retrieval tasks. Memory \& Cognition, 28, 1165-1172.

Voss, J. L., \& Paller, K. A. (2006). Fluent conceptual processing and explicit memory for faces are electrophysiologically distinct. Journal of Neuroscience, 26, 926-933.

Wilding, E. L. (2000). In what way does the parietal ERP $\mathrm{old} / \mathrm{new}$ effect index recollection? International Journal of Psychophysiology, 35, 81-87.

Wixted, J. T. (2007). Dual-process theory and signal-detection theory of recognition memory. Psychological Review, 114, $152-176$.

Wolk, D. A., Schacter, D. L., Lygizos, M., Sen, N. M., Chong, H., Holcomb, P. J., et al. (2007). ERP correlates of Remember/ Know decisions: Association with the late posterior negativity. Biological Psychology, 75, 131-135.

Woodruff, C. C., Hayama, H. R., \& Rugg, M. D. (2006). Electrophysiological dissociation of the neural correlates of recollection and familiarity. Brain Research, 1100, 125-135.

Yonelinas, A. P. (1994). Receiver-operating characteristics in recognition memory: Evidence for a dual-process model. Journal of Experimental Psychology: Learning, Memory, and Cognition, 20, 1341-1354.

Yonelinas, A. P. (1997). Recognition memory ROCs for item and associative information: The contribution of recollection and familiarity. Memory \& Cognition, 25, 747-763.

Yonelinas, A. P. (1999). The contribution of recollection and familiarity to recognition and source-memory judgments: A formal dual-process model and an analysis of receiver operating characteristics. Journal of Experimental Psychology: Learning, Memory, and Cognition, 25, 1415-1434.

Yonelinas, A. P. (2001a). Components of episodic memory: The contribution of recollection and familiarity. Philosophical Transactions of the Royal Society of London, Series B, Biological Sciences, 356, 1363-1374.

Yonelinas, A. P. (2001b). Consciousness, control, and confidence: The 3 Cs of recognition memory. Journal of Experimental Psychology: General, 130, 361-379.

Yonelinas, A. P. (2002). The nature of recollection and familiarity: A review of 30 years of research. Journal of Memory and Language, 46, 441-517.

Yonelinas, A. P., Kroll, N. E. A., Dobbins, I., Lazzara, M., \& Knight, R. T. (1998). Recollection and familiarity deficits in amnesia: Convergence of remember-know, process dissociation, and receiver operating characteristic data. Neuropsychology, 12, 323-339.

Yonelinas, A. P., \& Parks, C. M. (2007). Receiver operating characteristics (ROCs) in recognition memory. Psychological Bulletin, 133, 800-832.

Yonelinas, A. P., Quamme, J. R., Widaman, K. F., Kroll, N. E., Sauve, M. J., \& Knight, R. T. (2004). Mild hypoxia disrupts recollection, not familiarity. Cognitive, Affective $\mathcal{E}$ Behavioral Neuroscience, 4, 393-400; discussion 401-406. Yonelinas, A. P., Widaman, K., Mungas, D., Reed, B., Weiner, M. W., \& Chui, H. C. (in press). Memory in the aging brain: Doubly dissociating the contribution of the hippocampus and entorhinal cortex. Hippocampus.

Yovel, G., \& Paller, K. A. (2004). The neural basis of the butcher-on-the-bus phenomenon: When a face seems familiar but is not remembered. Neuroimage, 21, 789-800. 